\title{
Effect of the Oxidation of SH Groups on the Stability of Carp Myosin during Ice Storage
}

\author{
Warangkana Sompongse, Yoshiaki Itoh, Satoshi Nagamachi, ${ }^{\dagger}$ \\ and Atsushi Obatake \\ Department of Aquaculture, Faculty of Agriculture, Kochi University, \\ Nankoku, Kochi 783, Japan \\ (Received October 6, 1995)
}

\begin{abstract}
When carp myosin was stored in ice, a decrease in SH content and polymerization of myosin heavy chain (MHC) through SS bonding were observed along with a decrease in $\mathrm{Ca}^{2+}$-ATPase activity. By adding a chelating reagent (EDTA), the decrease in SH content and the polymerization were depressed. The addition of a reducing reagent (dithiothreitol) showed that the decrease in $\mathrm{Ca}^{2+}$-ATPase activity occurred without the polymerization by SS bonding. In contrast, the polymerization of $\mathrm{MHC}$ was greatly promoted in the presence of a metal ion $\left(\mathrm{Cu}^{2+}\right)$, whereas the decrease in $\mathrm{Ca}^{2+}$-ATPase activity was observed at the same level as without the metal ion. The addition of cryoprotectants (sorbitol, sucrose) showed a slight depressing effect on the decrease in $\mathrm{Ca}^{2+}$-ATPase activity and no influence on the polymerization of MHC.

It was concluded that the oxidation of $\mathrm{SH}$ groups in myosin along with the polymerization of MHC proceeded regardless of the conformational change in myosin head portion related to $\mathrm{Ca}^{2+}$. ATPase activity during ice storage. Therefore, it was suggested that the MHC polymerization during ice storage is due to SH groups on the myosin tail portion, even if myosin is free from actin.
\end{abstract}

Key words: myosin, polymerization, ice storage, $\mathrm{SH}$ groups

In the previous study, ${ }^{\text {l) }}$ we reported that the polymerization of myosin heavy chain by disulfide bonding is not due to the conformational change of myosin head portion related to $\mathrm{Ca}^{2+}$-ATPase activity during ice storage. Enhancement of $\mathrm{Ca}^{2+}$-ATPase activity by the modification of $\mathrm{SH}_{1}$ with NEM after ice storage of actomyosin showed that $\mathrm{SH}_{1}$ is not oxidized. ${ }^{2)}$ The increase in $\mathrm{Mg}^{2+}$ (EGTA)-ATPase activity during storage suggested that $\mathrm{SH}_{\mathrm{a}}$ on the myosin tail portion participates in the oxidation of actomyosin. ${ }^{2)}$

The covering of the $\mathrm{SH}_{1}$ region of myosin with actin has been suggested, since the modification of $\mathrm{SH}_{1}$ was performed under conditions to dissociate actomyosin to actin and myosin. ${ }^{3)}$ It can be concluded that $\mathrm{SH}_{1}$ might be oxidized when myosin is stored free from actin.

The main objective of the current study was to clarify the relationship between the oxidation of $\mathrm{SH}$ groups and the decrease in $\mathrm{Ca}^{2+}$-ATPase activity, when carp myosin is stored in ice. The results obtained from this experiment were compared with the previous data ${ }^{\mathrm{l})}$ on actomyosin, and the covering effect of actin against SH group oxidation of myosin was discussed.

\section{Materials and Methods}

\section{Myosin Preparation}

Myosin was prepared from carp Cyprinus carpio according to the procedure of Takashi et al. ${ }^{4)}$ With some modifications as follows. Minced dorsal muscle was washed with 4 volumes of phosphate buffer $(\mathrm{pH} 6.5, \mathrm{I}=0.1)$. Myosin was extracted with 3 volumes of $0.45 \mathrm{M} \mathrm{NaCl}-5 \mathrm{mM} \mathrm{ATP}-1 \mathrm{~mm}$ $\mathrm{MgCl}_{2}$ containing the above phosphate buffer $(\mathrm{pH}$ 6.5) for $4 \mathrm{~min}$. After purification by dilution-precipitation, myosin was adjusted to $0.6 \mathrm{M}$ in $\mathrm{NaCl}$ concentration and to approximately $0.4 \%$ in protein concentration. EDTA, DTT, $\mathrm{CuCl}_{2} \cdot 2 \mathrm{H}_{2} \mathrm{O}$, sorbitol, and sucrose were added separately to the myosin solution to obtain final concentrations of 5 $\mathrm{mm}, 5 \mathrm{~mm}, 1 \mathrm{ppm}, 10 \%$, and $20 \%$, respectively. The ice storage was performed under the same conditions as described in the previous paper. ${ }^{1)}$

\section{$\mathrm{Ca}^{2+}-$ ATPase Activity}

The same procedure as described in the previous paper was carried out.")

\section{SH Group Content}

$\mathrm{SH}$ content was measured in a solution of $8 \mathrm{M}$ urea- $2 \%$ SDS-10 mM EDTA-0.2 M Tris-HCl buffer ( $\mathrm{pH} \mathrm{6.8)} \mathrm{accord-}$ ing to the method of Ellman as described in the previous paper.")

\section{SDS-PAGE}

Ice-stored myosin was solubilized in $8 \mathrm{M}$ urea-2\% SDS$50 \mathrm{mM}$ phosphate buffer ( $\mathrm{pH} 6.8$ ) containing $0.2 \mathrm{mM}$ NEM, then the method of Weber and Osborn was performed as described in the previous paper. ${ }^{1}$

\footnotetext{
${ }^{\dagger}$ Present address: Hayashikane Sangyo Co., Ltd., Yamaguchi 750, Japan. Abbreviations: DTT, Dithiothreitol; EDTA, Ethylenediaminetetraacetic acid; MHC, Myosin Heavy Chain; MSH, 2-Mercaptoethanol; NEM, N-Ethylmaleimide; SDS-PAGE, Sodium Dodecyl Sulphate-Polyacrylamide Gel Electrophoresis; $\mathrm{SH}_{2}$, one sulfhydryl group on myosin tail region responsible for the activation of $\mathrm{Mg}^{2+}-\mathrm{ATPase}$; $\mathrm{SH}_{1}$, one reactive sulhydryl group on myosin head region related to $\mathrm{Ca}^{2+}$-ATPase activity.
} 


\section{Results and Discussion}

\section{Effect of a Chelating Reagent}

Changes in total SH content of carp myosin stored in the absence and presence of EDTA are shown in Fig. 1. Almost no change in the $\mathrm{SH}$ groups throughout ice storage was observed in the EDTA-containing sample, while in the absence of EDTA, the amount of SH groups slightly decreased. These results coincide with the effect of EDTA on the stability of SH groups of rabbit and trout myosins during storage at $0^{\circ} \mathrm{C}$, reported by Buttkus. ${ }^{5}$

SDS-PAGE patterns of myosin stored showed a decrease in MHC and production of higher molecular weight substances than MHC during the ice storage (Fig. 2). The major substance just above MHC was MHC dimer. The MHC dimer and the polymer (larger substances than MHC dimer) might be formed by SS bonding between MHC subunits, because in the electrophoretogram of the SDS-PAGE samples after reduction with $10 \%$ $\mathrm{MSH}$, the MHC dimer and the polymer were not ob-

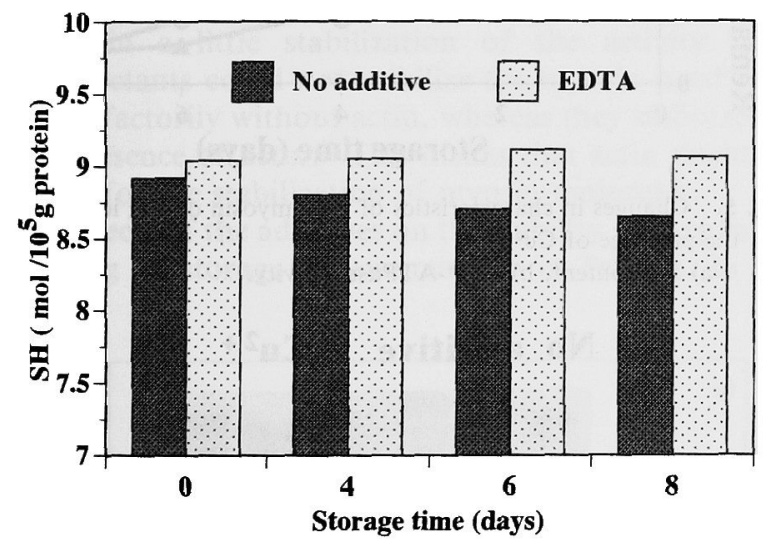

Fig. 1. Changes in SH content of carp myosin during ice storage in the presence of EDTA.

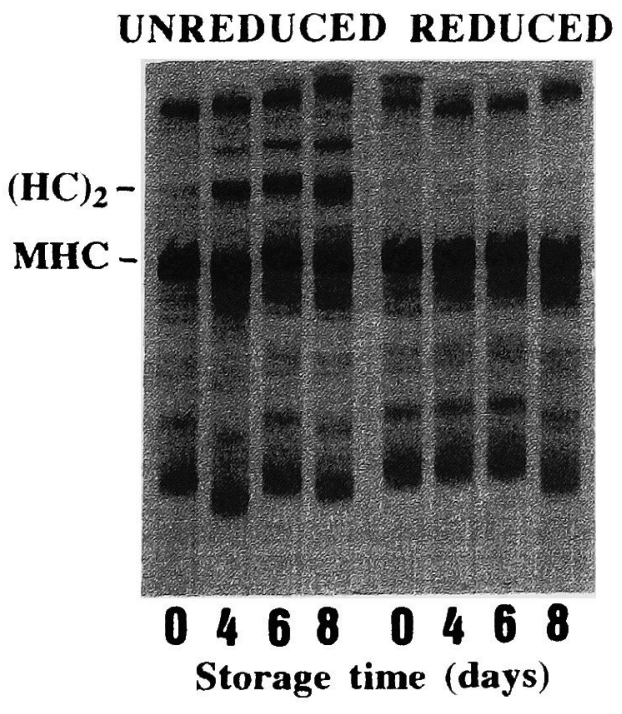

Fig. 2. SDS-PAGE patterns of carp myosin stored in ice. MHC: myosin heavy chain, $(\mathrm{HC})_{2}$ : dimer of MHC. served, and a similar amount of MHC was detected in all samples. After 8 days of storage, not only the amount of dimer but also that of the polymer increased (Fig. 3a).

Behaviors of MHC, dimer, and polymer during ice storage in the presence of EDTA are shown in Fig. 3b. The amount of the dimer and polymer formed through SS bonding was slightly suppressed in the presence of EDTA. The addition of EDTA was reported to decrease the rate of aggregation during incubation of rabbit myosin at $25^{\circ} \mathrm{C}^{6}$ and to suppress the polymerization of MHC through SS bonding during heating at $80^{\circ} \mathrm{C}^{7,8)}$ owing to the chelating of heavy metals. Based on these two reports, it was suggested that oxidation of SH groups of myosin did not occur in the presence of EDTA during ice storage because of its chelating action. A metal ion was considered to be
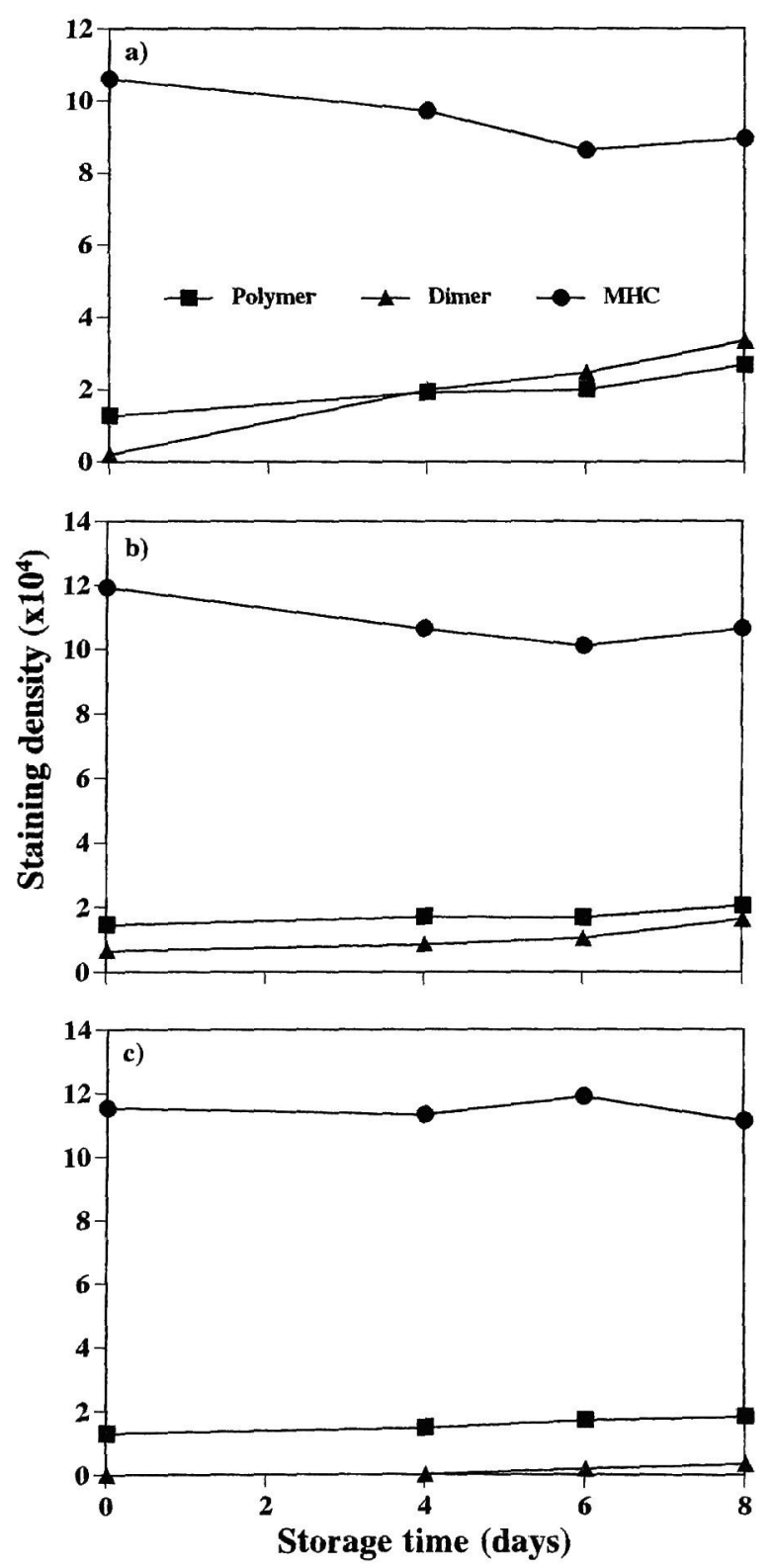

Fig. 3. Behavior of MHC, dimer and polymer of carp myosin during ice storage.

a) No additives, b) In the presence of EDTA, c) In the presence of DTT. 
necessary for the polymerization of MHC through SS bonding. In other words, the polymerization of MHC is mediated by the oxidation of SH groups between MHC subunits catalyzed by metal ions.

\section{Effect of a Reducing Reagent}

Further experiments were conducted to examine whether $\mathrm{Ca}^{2+}$-ATPase activity changes when disulfide formation was inhibited by the addition of a reducing agent, DTT.

The dimer formation through SS bonding did not occur in the presence of DTT, as shown in Fig. 3c. $\mathrm{Ca}^{2+}$-ATPase activity of myosin stored without DTT decreased enormously during the first four days, followed by a gradual decrease throughout the storage period (Fig. 4). Myosin in the presence of DTT also showed the same pattern of decrease in $\mathrm{Ca}^{2+}$-ATPase activity as in the absence of DTT. Based on these results, it was clarified that $\mathrm{Ca}^{2+}$ ATPase activity decreases even though the oxidation of $\mathrm{SH}$ groups is inhibited. $\mathrm{Ca}^{2+}$-ATPase activity of myosin decreased faster compared with actomyosin as already reported. ${ }^{1)}$ This difference is compatible with the results on the thermo-stability of myosin, reported by Murozuka et $a l .{ }^{9)}$ Therefore, the absence of actin might labilize the myosin head portion, which is the region related to $\mathrm{Ca}^{2+}$. ATPase activity.

\section{Effect of a Copper Ion}

To promote the oxidation of SH groups, $1 \mathrm{ppm}$ of $\mathrm{CuCl}_{2}$, a catalyst, was added to the myosin solution and the changes described above were followed during ice storage.

SH content decreased gradually during ice storage without $\mathrm{Cu}^{2+}$, as shown in Fig. 5a. The addition of $\mathrm{Cu}^{2+}$ resulted in a rapid decrease in the amount of SH groups. These results are in agreement with the report of Buttkus. ${ }^{5}$ During storage in the presence of $\mathrm{Cu}^{2+}$, greater amounts of the MHC dimer and the polymer were formed through SS bonding, which corresponded with a quick disappearance of MHC (Fig. 6).

Figure $5 \mathrm{~b}$ shows the changes in $\mathrm{Ca}^{2+}$-ATPase activity of myosin stored with and without $\mathrm{Cu}^{2+}$. Whether $\mathrm{Cu}^{2+}$ was added or not, the activities decreased in the same manner. These decrease patterns were the same as that of myosin

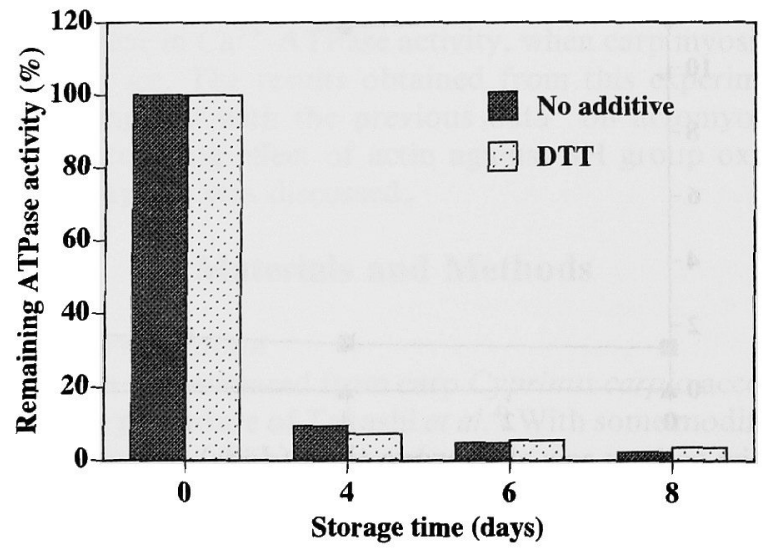

Fig. 4. Changes in $\mathrm{Ca}^{2+}$-ATPase activity of carp myosin during ice storage in the presence of DTT.

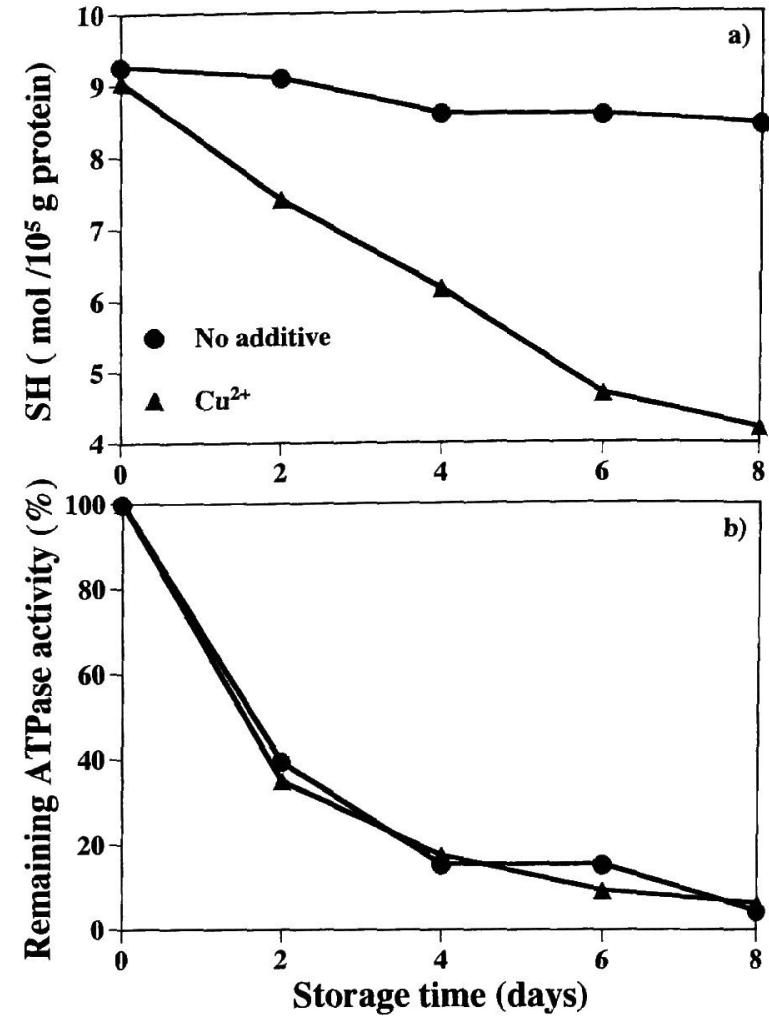

Fig. 5. Changes in characteristics of carp myosin during ice storage in the presence of $\mathrm{Cu}^{2+}$.

a) $\mathrm{SH}$ content, b) $\mathrm{Ca}^{2+}$-ATPase activity.

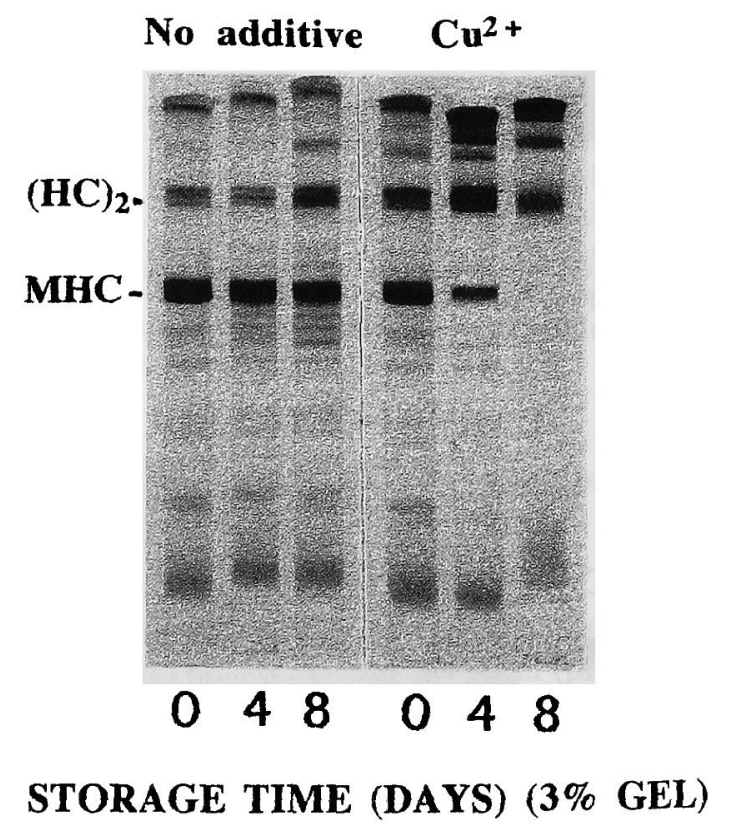

Fig. 6. SDS-PAGE patterns of carp myosin stored in ice in the presence of $\mathrm{Cu}^{2+}$.

MHC: myosin heavy chain, $(\mathrm{HC})_{2}$ : dimer of $\mathrm{MHC}$.

stored in the presence of DTT (Fig. 4), indicating that the decrease in $\mathrm{Ca}^{2+}$-ATPase activity occurred regardless of $\mathrm{SH}$ oxidation. The observation presented here supported the previous conclusion that the decrease in $\mathrm{Ca}^{2+}$-ATPase activity of myosin is not due to the oxidation of $\mathrm{SH}$ 
groups. However, this observation disagrees with the reports by Buttkus ${ }^{5)}$ and Hamada et al. ${ }^{10)}$ that the oxidation is associated with a decrease in $\mathrm{Ca}^{2+}$-ATPase activity during ice storage. The reason for the discrepancy cannot yet be explained.

\section{Effects of Cryoprotectants}

Sorbitol and sucrose, known as cryoprotectants, were added to the myosin solution, expecting to stabilize the myosin molecule during ice storage.

Changes in SH content of myosin in the absence and presence of cryoprotectants are shown in Fig. 7. Regardless of the addition of cryoprotectants, SH content decreased continuously during ice storage.

SDS-PAGE patterns in Fig. 8 show that the dimer and the polymer of MHC were formed through SS bonding even in the presence of sucrose, supporting the behavior of the decrease in SH content in the absence and presence of sucrose. A similar trend of polymerization behavior of MHC on SDS-PAGE patterns was also obtained from the sample stored in the presence of sorbitol (data not shown).

$\mathrm{Ca}^{2+}$-ATPase activity decreased significantly in the first 2 or 4 days (Fig. 9). The addition of cryoprotectants resulted in a little stabilization of the activity. The cryoprotectants could not stabilize the myosin head portion satisfactorily without actin, whereas they stabilized it in the presence of actin. ${ }^{1)}$ This means that actin might be essential for the stabilization of myosin molecules.

The effects of the additives on the stability of carp myosin during ice storage are summarized in Table 1. In the

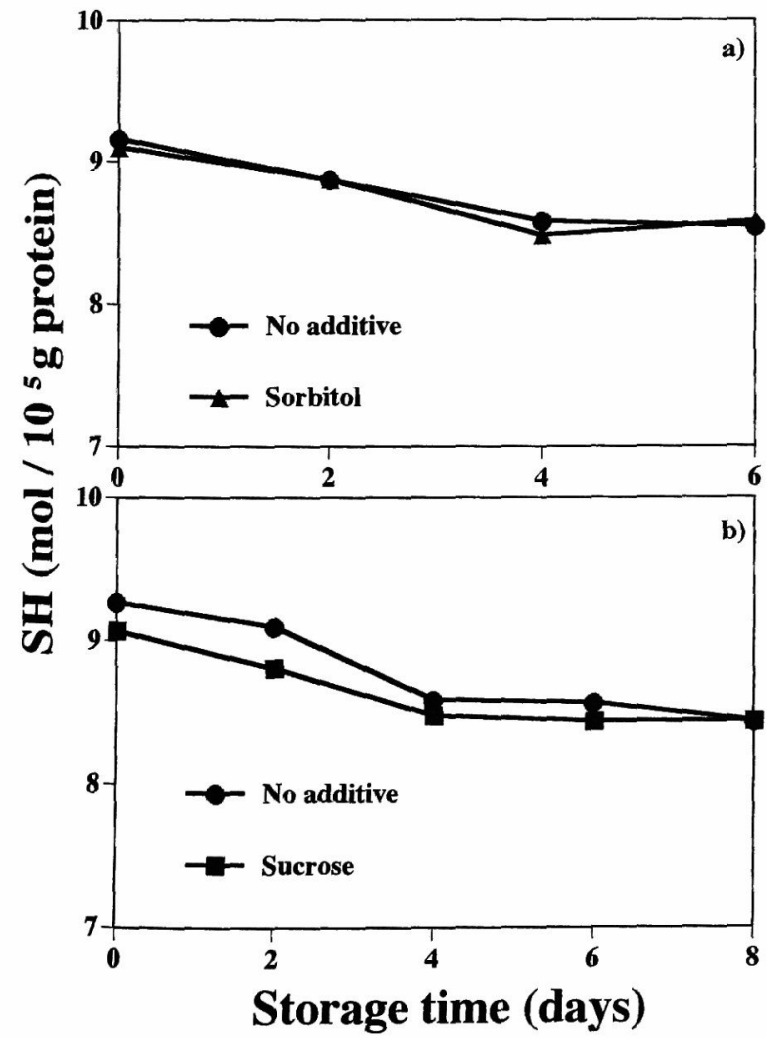

Fig. 7. Changes in SH content of carp myosin during ice storage in the presence of cryoprotectants.

a) In the presence of sorbitol, b) In the presence of sucrose.

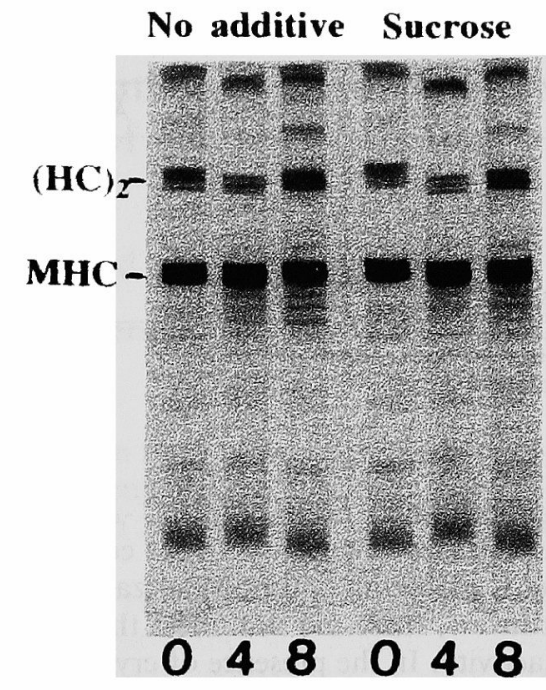

\section{STORAGE TIME (DAYS) (3\% GEL)}

Fig. 8. SDS-PAGE patterns of carp myosin stored in ice in the presence of sucrose.

MHC: myosin heavy chain, $(\mathrm{HC})_{2}$ : dimer of MHC.

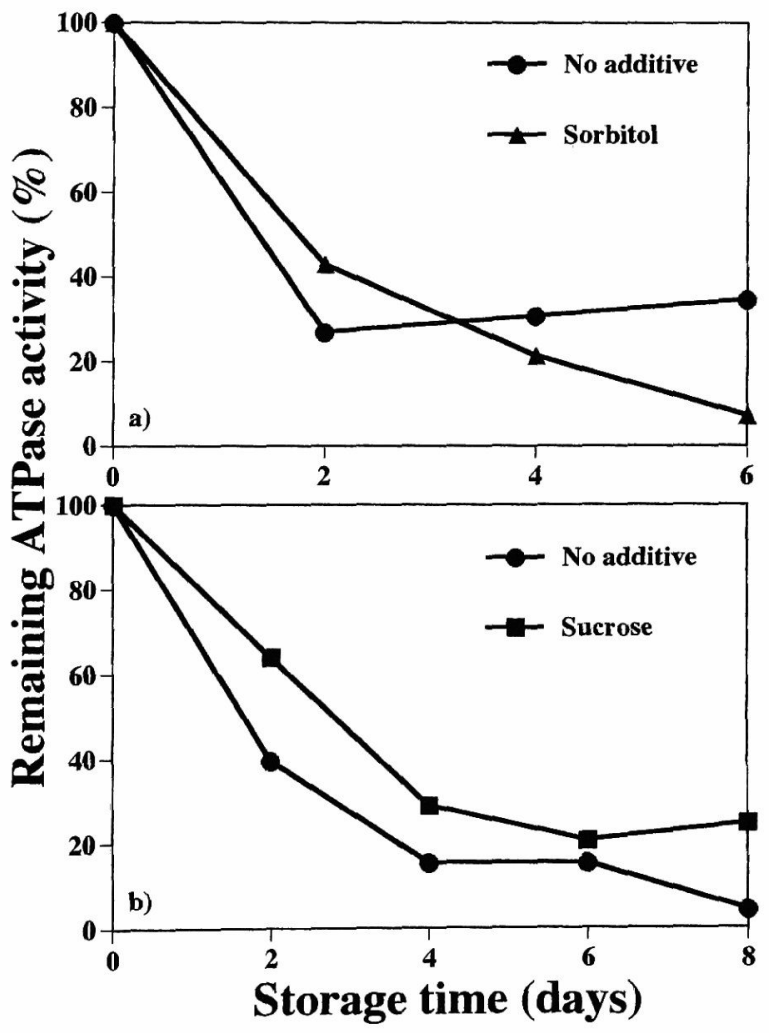

Fig. 9. Changes in $\mathrm{Ca}^{2+}$-ATPase activity of carp myosin during ice storage in the presence of cryoprotectants.

a) In the presence of sorbitol, b) In the presence of sucrose.

control sample, SH content of myosin decreased, accompanied by the polymerization of MHC through SS bonding and by the decrease in $\mathrm{Ca}^{2+}$-ATPase activity. In the presence of EDTA, neither oxidation of SH groups nor polymerization occurred owing to the chelating action of EDTA. In the presence of DTT, the polymerization 
Table 1. Effects of additives on the stability of carp myosin during ice storage

\begin{tabular}{|c|c|c|c|}
\hline Additives & Oxidation & Polymerization & ATPase-activity \\
\hline None & + & + & $\downarrow$ \\
\hline EDTA & - & - & \\
\hline DTT & & - & $\downarrow$ \\
\hline $\mathrm{Cu}^{2+}$ & $+t+$ & $+t+$ & $\downarrow$ \\
\hline Cryoprotectants & + & + & $\downarrow$ \\
\hline
\end{tabular}

+ : induced $++t$ induced strongly

- : inhibited $t$ : decreased

through SS bonding did not occur, but $\mathrm{Ca}^{2+}$-ATPase activity decreased in the same manner as in the control. In the presence of $\mathrm{Cu}^{2+}$, oxidation and polymerization were enhanced. Nevertheless, these did not affect the decrease in $\mathrm{Ca}^{2+}$-ATPase activity. In the presence of cryoprotectants, $\mathrm{Ca}^{2+}$-ATPase activity was stabilized significantly, but oxidation and polymerization were not affected compared with those in the control.

In conclusion, whether oxidation was enhanced or inhibited, $\mathrm{Ca}^{2+}$-ATPase activity decreased during ice storage. In other words, the decrease in $\mathrm{Ca}^{2+}$-ATPase activity is not related to the oxidation of $\mathrm{SH}$ groups, even in the absence of actin. This greatly supports the previous suggestion. ${ }^{1)}$ Therefore, polymerization of MHC through SS bonding could be attributed to the oxidation of SH groups on the myosin tail portion, not on the myosin head portion. The portion of SH groups in myosin responsible for the polymerization of $\mathrm{MHC}$ will be reported in the next paper.

\section{References}

1) W. Sompongse, Y. Itoh, and A. Obatake: Effect of cryoprotectants and a reducing reagent on the stability of actomyosin during ice storage. Fisheries Sci., 62, 73-79 (1996).

2) W. Sompongse, Y. Itoh, and A. Obatake: Role of $\mathrm{SH}_{\mathrm{a}}$ in the polymerization of myosin heavy chain during ice storage of carp actomyosin. Fisheries Sci., 62, 110-113 (1996).

3) T. Yamashita, M. Kobayashi, and T. Horigome: The sulfhydryl groups involved in the active site of myosin $B$ adenosinetriphosphatase 1. Relationship of sulfhydryl group responsible for $\mathrm{Mg}^{2+}$-ATPase activation to the $\mathrm{S}_{1}$ and $\mathrm{S}_{2}$ groups. J. Biochem., 77, 1037-1046 (1975).

4) R. Takashi, K. Arai, and T. Saito: Studies on muscular proteins of fish-I. Preparation of myosin from carp muscle. Nippon Suisan Gakkaishi, 36, 165-168, (1970).

5) H. Buttkus: The sulfhydryl content of rabbit and trout myosins in relation to protein stability. Can. J. Biochem., 49, 97-107 (1971)

6) S. Lowey and A. Holtzer: The aggregation of myosin. Amer. Chem. Soc., 81, 1378-1383 (1959)

7) A. Kishi, Y. Itoh, and A. Obatake: The polymerization of protein through disulfide bonding during the heating of carp myosin. Nippon Suisan Gakkaishi, 61, 75-80 (1995).

8) A. Kishi, Y. Itoh, and A. Obatake: Effect of buffers on the polymerization of protein through disulfide bonding during heating of myosin from carp muscle. Nippon Suisan Gakkaishi, 61, 380-386 (1995).

9) T. Murozuka, R. Takashi, and K. Arai: Relative thermostabilities of $\mathrm{Ca}^{2+}$ ATPase of myosin and actomyosin from tilapia and rabbit. Nippon Suisan Gakkaishi, 42, 57-63 (1976).

10) I. Hamada, K. Tsuji, T. Nakayama, and E. Niwa: Oxidative denaturation of actomyosin. Nippon Suisan Gakkaishi, 43, 1105-1109 (1977). 